\title{
New Herbig-Haro objects in the L1617 and L1646 dark clouds
}

\author{
H. Wang ${ }^{1,2}$, B. Stecklum ${ }^{3}$, and Th. Henning ${ }^{2}$ \\ 1 Purple Mountain Observatory, Academia Sinica, Nanjing 210008, PR China \\ e-mail: hcwang@pmo.ac.cn \\ 2 Max-Planck-Institut für Astronomie, Königstuhl 17, 69117 Heidelberg, Germany \\ e-mail: henning@mpia-hd.mpg.de \\ 3 Thüringer Landessternwarte Tautenburg, Sternwarte 5, 07778 Tautenburg, Germany \\ e-mail: stecklum@tls-tautenburg.de
}

Received 26 January 2005 / Accepted 25 February 2005

\begin{abstract}
Optical imaging towards L1617 and L1646 revealed three new Herbig-Haro (HH) objects, HH 182, 439, and 866. Spectroscopic observations of HH 182 A and 439 A confirmed their HH object nature. Molecular hydrogen $v=1-0$ S(1) narrow band imaging revealed three $\mathrm{H}_{2}$ emission features in the $\mathrm{HH} 182$ region which coincide with the optical emission. Based on the position angles of the different parts of the HH 111 flow and that of HH 182, HH 182 may be the outermost southeastern part of the giant HH 111 flow. One deeply embedded star is revealed in our near-infrared imaging of the HH 439 region. HH 439 A and the associated bow shock are probably driven by the newly detected embedded star. HH 439 B-D are probably driven by the Herbig AeBe star candidate GSC 04794-00827 (IRAS 06045-0554). The embedded source IRAS 06046-0603 is identified to be the exciting source of $\mathrm{HH} 866$.
\end{abstract}

Key words. Herbig-Haro objects - ISM: jets and outflows - ISM: individual objects: L1617 - ISM: individual objects: L1646 stars: formation

\section{Introduction}

Herbig-Haro objects are small emission nebulae with characteristic spectra (a recent catalogue of $\mathrm{HH}$ objects is available at http://casa.colorado.edu/hhcat/ which is maintained by B. Reipurth). They are produced as supersonic $\left(v \sim 300 \mathrm{~km} \mathrm{~s}^{-1}\right)$ mass outflows from young stellar objects (YSOs) collide with the surrounding medium or when faster ejecta catch up with the slower ones produced in previous ejection episodes. Important information on mass outflows from YSOs, such as the mass-loss rates and momentum rates, can be obtained from the study of HH objects (Hartigan et al. 1994; Bacciotti et al. 1995). The bipolar or highly collimated morphology of some HH flows, e.g., HH 1/2 and HH 111, have lead to the identification of deeply embedded stars at the very early stage of star formation (Pravdo et al. 1985; Reipurth et al. 1993). It has been established that mass outflows from YSOs are driven by mass accretion onto central stars (Shu et al. 2000; Köenig et al. 2000). Therefore, HH flows contain important information about mass accretion of YSOs. Indeed, a lot of HH flows (e.g. HH 111 and 212) are found to be episodic, implying that mass accretion is also likely a time-dependent process (Reipurth et al. 1997b; Zinnecker et al. 1998). In particular, dozens of giant HH flows of parsec-scales have been found (see, e.g., Eislöffel \& Mundt 1997; Reipurth et al. 1997a). Some of these giant HH flows, e.g., HH 34 and the PV Cep flows, exhibit S shape symmetry with respect to the driving sources, showing that the axes of accretion disks were precessing in the past $\sim 10^{4} \mathrm{yr}$ (Devine et al. 1997; Reipurth et al. 1997a).

Mass outflows from YSOs have also been observed at other wavelengths, e.g., $\mathrm{H}_{2}$ emission in the near-infrared and molecular outflows at millimeter wavelengths (see Bachiller 1996; Eislöeffel 1997). It is generally believed that momentum transfer from the high-velocity ejecta to the surrounding medium gives rise to the near-infrared $\mathrm{H}_{2}$ emission and molecular outflows observed around YSOs (Hartigan et al. 2000). However, the details of these processes still remain unknown. Multiwavelength studies of mass outflows will provide hints to the mechanisms at work in these processes.

In order to investigate mass outflows from YSOs, we have conducted a large-scale HH object survey of nearby star-forming regions (see Yan et al. 1998; Wang et al. 2001, 2003). Our survey fields include the L1617 and L1646 dark clouds. The L1617 dark cloud is located in the Orion B molecular cloud complex at a distance of $460 \mathrm{pc}$ from the Sun. It consists of three condensations of cometary shape (Bally et al. 1991). Nine HH objects, i.e., HH 110-113 (Reipurth \& Olberg 1991; Reipurth et al. 1997a), 121 (Gredel \& Reipurth 1993), 270 (Reipurth et al. 1996), 311 (Reipurth et al. 1997a), and 490-491 (Noriega-Crespo et al. 2002), as well as several YSOs have been discovered in the cloud (Rodríguez et al. 1998; Dent et al. 1998). The L1646 dark cloud is located towards the Monoceros R2 nebula at a distance 
Table 1. Log of imaging observations.

\begin{tabular}{lllll}
\hline \hline Field & Filter & Telescope & Exposure & Date \\
\hline L1617 & {$[$ BATC09] } & BAO 0.6/0.9 m & $4 \times 300 \mathrm{~s}$ & $1996 \mathrm{Feb}$. \\
& {$[$ BATC10] } & BAO 0.6/0.9 m & $4 \times 300 \mathrm{~s}$ & $1996 \mathrm{Feb}$. \\
& {$[\mathrm{S} \mathrm{II}]$} & BAO 0.6/0.9 m & $3 \times 1200 \mathrm{~s}$ & $1997 \mathrm{Mar}$. \\
L1646 & {$[$ BATC09] } & BAO 0.6/0.9 m & $3 \times 480 \mathrm{~s}$ & $1996 \mathrm{Feb}$. \\
& {$[$ BATC10] } & BAO 0.6/0.9 m & $3 \times 480 \mathrm{~s}$ & $1996 \mathrm{Feb}$. \\
& {$[\mathrm{S} \mathrm{II}]$} & BAO 0.6/0.9 m & $6 \times 600 \mathrm{~s}$ & 1996 Dec. \\
& {$[\mathrm{S} \mathrm{II}]$} & TLS $1.34 / 2.00 \mathrm{~m}$ & $2 \times 1200 \mathrm{~s}$ & $2002 \mathrm{Mar}$. \\
& $\mathrm{H}_{\alpha}$ & TLS $1.34 / 2.00 \mathrm{~m}$ & $1 \times 600 \mathrm{~s}$ & $2002 \mathrm{Mar}$. \\
& $\mathrm{I}$ & TLS $1.34 / 2.00 \mathrm{~m}$ & $2 \times 180 \mathrm{~s}$ & $2002 \mathrm{Mar}$. \\
\hline
\end{tabular}

of 830 pc (Herbst \& Racine 1976). Three HH objects, HH 271-273 (Carballo \& Eiroa 1992), as well as several pre-mainsequence stars (Herbig \& Bell 1988) have been discovered in the cloud.

In this paper we report the discovery of three new $\mathrm{HH}$ objects, HH 182, 439, and 866 in the L1617 and L1646 dark clouds. The observations are described in Sect. 2 and the results are presented in Sect. 3. We discuss the results in Sect. 4 and our conclusions are summarized in Sect. 5.

\section{Observations}

$\mathrm{HH} 182$ and 439 were first discovered as $\mathrm{HH}$ object candidates when the intermediate-band [BATC09] and [BATC10] filters were used in our HH object survey of the L1617 and L1646 dark clouds. These filters have a bandpass of 480 and $300 \AA$, respectively, with their central wavelengths at 6660 and $7050 \AA$. The bandwidth of the [BATC09] filter covers most of the characteristic emission lines of $\mathrm{HH}$ objects, such as the [N II] $\lambda \lambda 6548 / 6583, \mathrm{H}_{\alpha}$ and the [S II] $\lambda \lambda 6717 / 6731$ emission lines, while the [BATC10] filter covers no strong emission lines of $\mathrm{HH}$ objects, therefore being used to measure the continuum emission. The $\mathrm{HH}$ nature of these objects was subsequently confirmed by follow-up narrow band imaging through a [S II] filter $\left(\lambda_{\mathrm{c}}=6725 \AA, \Delta \lambda=50 \AA\right)$. The telescope used for these observations is the $\mathrm{f} / 360 / 90 \mathrm{~cm}$ Schmidt telescope at Xinglong Station of Beijing Astronomical Observatory (BAO), which is equipped with a $2048 \times 2048$ Aerospace Ford CCD. The pixel size is $15 \mu \mathrm{m}$, corresponding to a resolution of $1^{\prime \prime}$.67/pixel, and the field of view is $57^{\prime} \times 57^{\prime}$ (Fan et al. 1996). The L1646 region was further observed through the narrow band [S II] and $\mathrm{H}_{\alpha}$ filters using the $1.34 / 2.00 \mathrm{~m}$ Schmidt telescope at Thüringer Landessternwarte Tautenburg (TLS), Germany. This telescope is equipped with a $2048 \times 2048$ SITe CCD camera which has a field of view of $42^{\prime} \times 42^{\prime}$. The pixel size is $24 \mu \mathrm{m}$, corresponding to a resolution of 1 '.23/pixel. The effective field of view, limited by the dimensions of the filters used in our observations, is around $\sim 37^{\prime} \times 37^{\prime}$. The journal of the imaging observations is given in Table 1 .

The images are bias subtracted, dome flat normalized, bad pixels removed, and then are combined using the $\mathrm{IRAF}^{1}$ package. The seeing during the BAO observations was $\sim 4$.'0 and $\sim 3^{\prime \prime}$. 0 during the TLS observations.

${ }^{1}$ IRAF is distributed by the National Optical Astronomy Observatories, which are operated by the Association of Universities

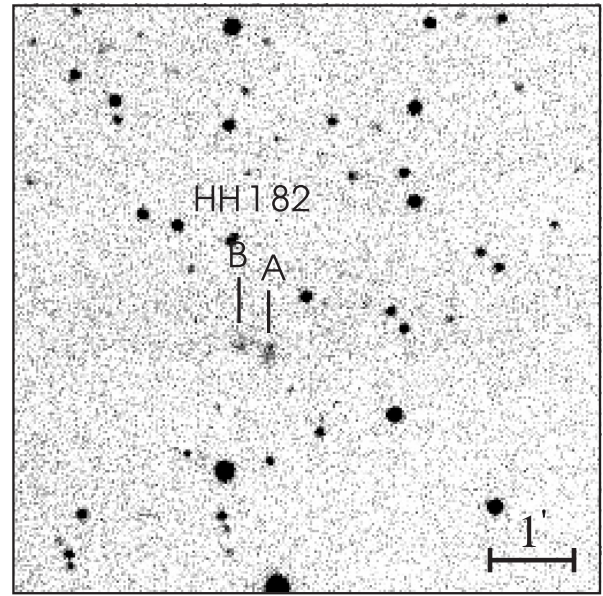

Fig. 1. [S II] image of HH 182. The field is $7^{\prime} \times 7^{\prime}$; north is up, east is to the left, and the scale is indicated.

Low-dispersion spectroscopic observations of $\mathrm{HH} 182 \mathrm{~A}$ and 439 A were made with the BAO $2.16 \mathrm{~m}$ telescope using a Cassegrain spectrograph on 1999 Dec. 4-5. The grating used is $100 \AA \mathrm{mm}^{-1}$ and the slit width is 2 '. 0 . The resulting spectral resolution is $\sim 9 \AA$. Using the offset to a nearby star, the slit is centered on the [S II] emission maximum and is oriented along the north-south direction. The total exposure for both $\mathrm{HH}$ $182 \mathrm{~A}$ and $439 \mathrm{~A}$ is $90 \mathrm{~min}$. The spatial extents for HH $182 \mathrm{~A}$ and $439 \mathrm{~A}$ where the spectra are extracted are $16^{\prime \prime}$ and $14^{\prime \prime}$, respectively.

The near-infrared imaging observations toward the $\mathrm{HH}$ 182 and 439 regions were carried out on 1999 November 21 and 25 using the $1.88 \mathrm{~m}$ telescope at the Okayama Astronomical Observatory, Japan. The infrared camera OASIS (Yamashita et al. 1995), which is equipped with a near-infrared camera and multi-object spectrometer (NICMOS3) array, was used for the observations. The field of view of OASIS is $4.2 \times 4.2$ with a plate scale of $0.97 /$ pixel. Dithered $J, H, K^{\prime}$ $(2.16 \mu \mathrm{m})$ and $\mathrm{H}_{2} v=1-0 \mathrm{~S}(1)(2.12 \mu \mathrm{m})$ images were obtained. The total exposures toward the $\mathrm{HH} 182$ region in the $J H K^{\prime}$ and $\mathrm{H}_{2}$ bands are $100,80,100$, and $300 \mathrm{~s}$, respectively, and those toward the $\mathrm{HH} 439$ region are 100, 50, 50, and $300 \mathrm{~s}$. The weather conditions during the observations were good and the seeing was around 2 .'0. The images were dark subtracted, flat field normalized, sky frame subtracted, registered and then combined using the IRAF package. The flat field was constructed with two sets of dome flat frames taken by switching an illuminating lamp on and off. The sky frame was obtained by median filtering the target images. The standard stars used for photometry are AS 10 and 13 (Hunt et al. 1998).

\section{Results}

In the L1617 dark cloud one new HH object, HH 182, was discovered in our optical images. The optical image of HH 182 in the $[\mathrm{S} \mathrm{II}]$ band is presented in Fig. 1. From Fig. 1 we can see that $\mathrm{HH} 182$ consists of 2 emission patches, here designated

for Research in Astronomy, Inc., under cooperative agreement with the National Science Foundation. 
Table 2. New Herbig-Haro objects.

\begin{tabular}{rccl}
\hline \hline Object & $\alpha(\mathrm{J} 2000)$ & $\delta(\mathrm{J} 2000)$ & Comments \\
\hline 182 A & 55409.8 & 23709 & Patch \\
B & 55411.2 & 23715 & Faint patch \\
439 A & 60654.7 & -55328 & Bright elongated patch \\
B & 60654.2 & -55431 & Bright knot \\
C & 60653.3 & -55418 & Knot \\
D & 60654.4 & -55454 & Knot \\
866 A & 60707.6 & -60337 & Bright knot \\
B & 60707.8 & -60350 & Faint patch \\
C & 60707.3 & -60327 & Faint patch \\
\hline
\end{tabular}

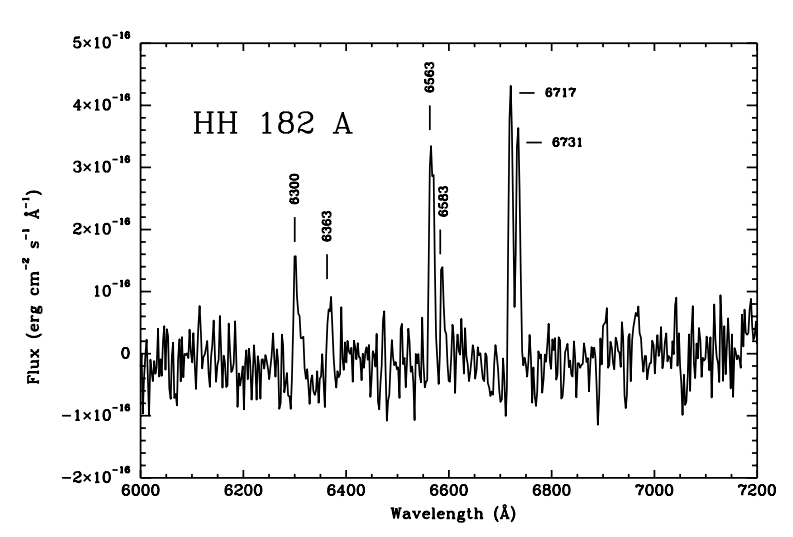

Fig. 2. Low dispersion spectrum of HH 182 A.

HH 182 A and B. Their coordinates, which are obtained using the GSC catalog to determine the plate solutions, are listed in Table 2. Our astrometry is accurate within 1 " both in right ascension and declination.

In Fig. 2 we present the low dispersion spectrum of HH 182 A. The observed line intensities, which are obtained through Gaussian fitting to the line profiles, are given in Table 3. As no extinction information of the region is available and the $\mathrm{H}_{\beta}$ emission is not detected in our spectrum of HH 182 A, we did not carry out extinction corrections to the observed line intensities. As HH 182 is located in the outskirts of L1617 (Bally et al. 1991) and the wavelengths listed in Table 3 cover a small range, the reddening should not have a significant effect on the line ratios. The rms noise in the spectrum of $\mathrm{HH}$ $182 \mathrm{~A}$ is around $3.0 \times 10^{-17} \mathrm{erg} \mathrm{cm}^{-2} \mathrm{~s}^{-1} \AA^{-1}$. From this value and the line intensity list in Table 3 , we estimated that the line ratio $[\mathrm{S} \mathrm{II}] \lambda \lambda(6717+6731) / \mathrm{H}_{\alpha}$ is $2.0 \pm 0.18$ for $\mathrm{HH} 182 \mathrm{~A}$, indicating that $\mathrm{HH} 182 \mathrm{~A}$ has a low excitation (Raga et al. 1996). The [S II] $\lambda 6717 / \lambda 6731$ intensity ratio for $\mathrm{HH} 182 \mathrm{~A}$ is $1.19 \pm$ 0.13 , which indicates an electron density in the range from 100 to $440 \mathrm{~cm}^{-3}$ (Osterbrock 1989).

In Fig. 3a we present the image of the $\mathrm{HH} 182$ region in the $\mathrm{H}_{2}$ band. The continuum-subtracted $\mathrm{H}_{2}$ image is presented in Fig. 3b. In Fig. 3b the contours of the optical [S II] emission of $\mathrm{HH} 182 \mathrm{~A}$ and B are superposed on the image. Three $\mathrm{H}_{2}$ emission patches can be seen from Figs. $3 \mathrm{a}-\mathrm{c}$. These $\mathrm{H}_{2}$ emission patches are labeled A-C in Figs. 3a and c. Patch A is bright and has an arc shape while patches $\mathrm{B}$ and $\mathrm{C}$ are rather weak.

In the L1646 dark cloud two new HH objects, HH 439 and 866 , were discovered in our optical images. The optical
Table 3. Emission-line ratios for HH 182 A and 439 A.

\begin{tabular}{lccl}
\hline \hline Object & Wavelength $(\AA)$ & Identification & Intensity \\
\hline HH 182 A & & & \\
& 6300 & O I & 58 \\
& 6363 & O I & 25 \\
& 6563 & $\mathrm{H}_{\alpha}$ & 100 \\
& 6583 & $\mathrm{~N}$ II & 30 \\
& 6717 & $\mathrm{~S}$ II & 108 \\
& 6731 & $\mathrm{~S}_{\text {II }}$ & 91 \\
HH 439 A & & & \\
& 6300 & $\mathrm{O}_{\text {I }}$ & 24 \\
& 6563 & $\mathrm{H}_{\alpha}$ & 100 \\
& 6583 & $\mathrm{~N}$ II & 20 \\
& 6717 & $\mathrm{~S} \mathrm{II}$ & 36 \\
& 6731 & $\mathrm{~S}_{\text {II }}$ & 31 \\
\hline
\end{tabular}

images of $\mathrm{HH} 439$ in the [S II] and $\mathrm{H}_{\alpha}$ bands are presented in Fig. 4. HH 439 is an emission complex consisting of patch, knots and diffuse emission (see Fig. 4). HH 439 A is a bright, elongated patch oriented in the direction of $\mathrm{PA}=70^{\circ}$ with a projected length of $\sim 15^{\prime \prime}$. HH $439 \mathrm{~B}-\mathrm{D}$ are emission knots. HH 439 A-D can be identified both in the [S II] and $\mathrm{H}_{\alpha}$ images although knots $\mathrm{B}-\mathrm{D}$ are rather weak in the $\mathrm{H}_{\alpha}$ image. The coordinates of HH 439 A-D are listed in Table 2. HH 439 A appears brighter in the $\mathrm{H}_{\alpha}$ image than in the [S II] image, indicating that it has a relatively high excitation degree. In contrast, $\mathrm{HH} 439$ B-D are much weaker in the $\mathrm{H}_{\alpha}$ image than in the [S II] image, indicating that they have a relatively low excitation degree. A bow shock consisting of $\mathrm{HH} 439 \mathrm{~A}$ and the diffuse emission to its northeast and southeast can be seen in the [S II] image. This bow shock is marked in Fig. 4a with a black curve.

The low dispersion optical spectrum of HH 439 A is presented in Fig. 5. The observed line intensities are given in Table 3. As in the case of $\mathrm{HH} \mathrm{182,} \mathrm{no} \mathrm{extinction} \mathrm{informa-}$ tion of the region is available and the $\mathrm{H}_{\beta}$ emission is not detected in our spectrum. Therefore we did not carry out extinction corrections to the observed line intensities. From our optical images we estimate that the reddening effect on the line intensity ratios is small. The rms noise in the spectrum is around $3.0 \times 10^{-17} \mathrm{erg} \mathrm{cm}^{-2} \mathrm{~s}^{-1} \AA^{-1}$. From this value and the line intensity list in Table 3 , we estimated that $[\mathrm{S}$ II $] \lambda \lambda(6717+6731) / \mathrm{H}_{\alpha}=0.67 \pm 0.04$ for $\mathrm{HH} 439 \mathrm{~A}$, indicating that $\mathrm{HH} 439$ A has a high excitation (Raga et al. 1996), consistent with the conclusion inferred from comparison of the [S II] and $\mathrm{H}_{\alpha}$ images. The [S II] $\lambda 6717 / \lambda 6731$ intensity ratio for $\mathrm{HH} 439 \mathrm{~A}$ is $1.16 \pm 0.04$, which indicates an electron density of $300 \pm 60 \mathrm{~cm}^{-3}$.

The [S II] and $\mathrm{H}_{\alpha}$ images of $\mathrm{HH} 866$ are shown in Fig. 6. From Fig. 6a, we can see that HH 866 consists of 3 components, HH 866 A-C. HH $866 \mathrm{~A}$ is a bright knot. HH $866 \mathrm{~B}$ and $\mathrm{C}$ are faint patches. In the $\mathrm{H}_{\alpha}$ image, only $\mathrm{HH} 866 \mathrm{~A}$ is detected and it is much fainter than in the [S II] image. A comparison of the [S II] and $\mathrm{H}_{\alpha}$ images shows that $\mathrm{HH} 866 \mathrm{~A}-\mathrm{C}$ have low excitations. The coordinates of $\mathrm{HH} 866 \mathrm{~A}-\mathrm{C}$ are listed in Table 2. 

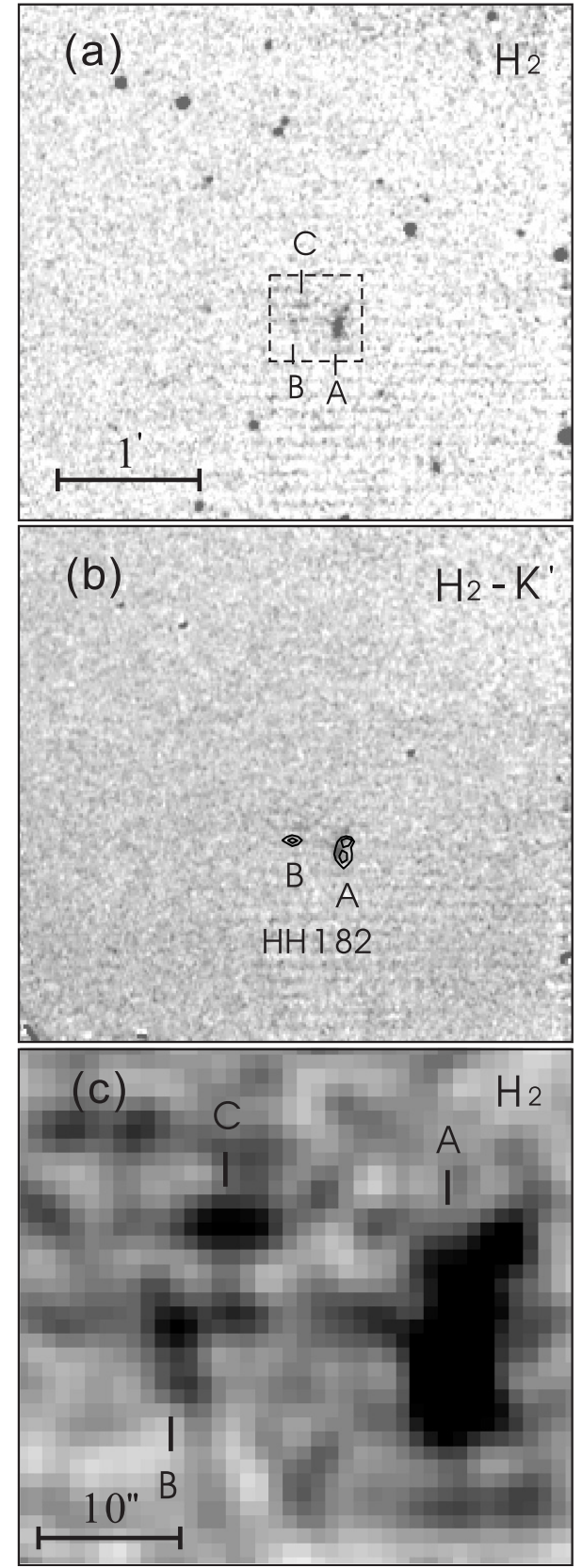

Fig. 3. $\mathrm{H}_{2}$ emission in the $\mathrm{HH} 182$ region, a) $\mathrm{H}_{2}$ emission plus the continuum; b) the net $\mathrm{H}_{2}$ emission; c) a zoom on the $\mathrm{HH} 182$ region within the dashed lines in a) to show the faint $\mathrm{H}_{2}$ emission patches $\mathrm{B}$ and $\mathrm{C}$. The fields in a) and b) are 3.9 $\times 3.6$; north is up, east is to the left. The optical [S II] emission of HH $182 \mathrm{~A}$ and B is shown as contours in panel $\mathbf{b}$ ). The first contour is $2 \sigma$ above the background and the second is $3 \sigma$.

\section{Discussions}

\section{1. $H H 182$}

From Fig. $3 b$ it can be seen that the optical emission of $\mathrm{HH}$ $182 \mathrm{~A}$ and $\mathrm{B}$ coincides well with the near-infrared $\mathrm{H}_{2}$ emission. Both the optical (see the contours in Fig. 3b) and the nearinfrared $\mathrm{H}_{2}$ emission images (see Fig. 3) show that $\mathrm{HH} 182 \mathrm{~A}$ has an arc shape facing towards the east. This morphology suggests that the HH 182 flow may be excited by a source located

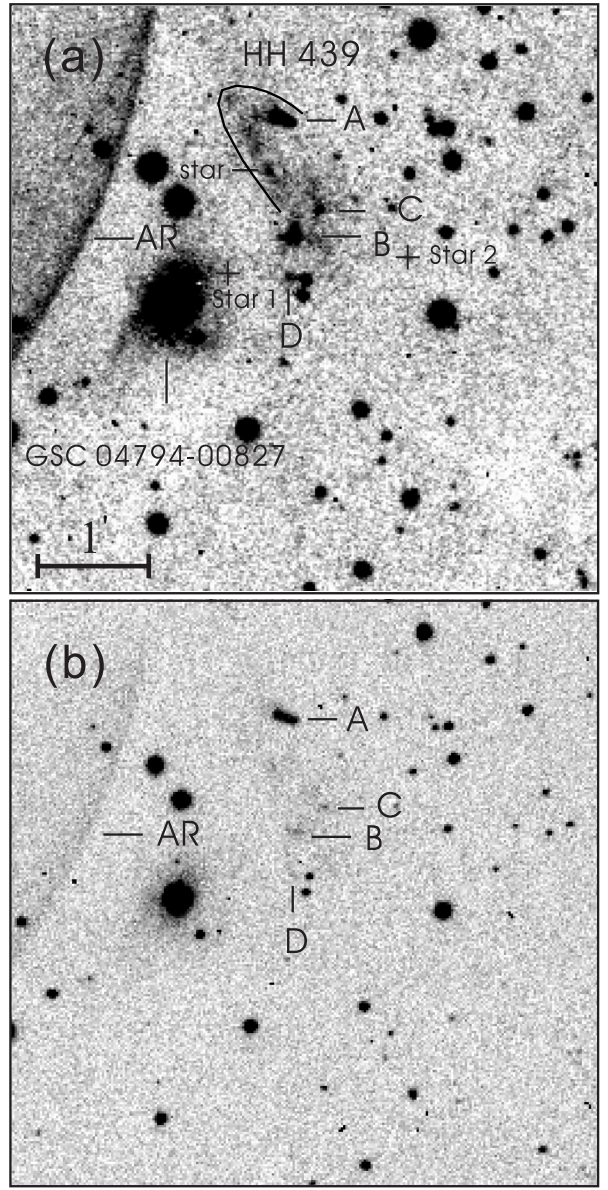

Fig. 4. Optical images of HH 439 taken with the TLS Schmidt telescope, a) in the [S II] band; b) in the $\mathrm{H}_{\alpha}$ band. The fields are $5 ! 3 \times 5 ! 3$; north is up, east is to the left. The scale is indicated in a). The nearby Herbig AeBe star, GSC 04794-00827, is indicated in a). The positions of the embedded stars 1 and 2 detected in our near-infrared imaging (see Sect. 4.2) are marked with plus signs. The artifacts in $\mathbf{a}$ ) and b) due to the reflection of a nearby bright star are marked with "AR".

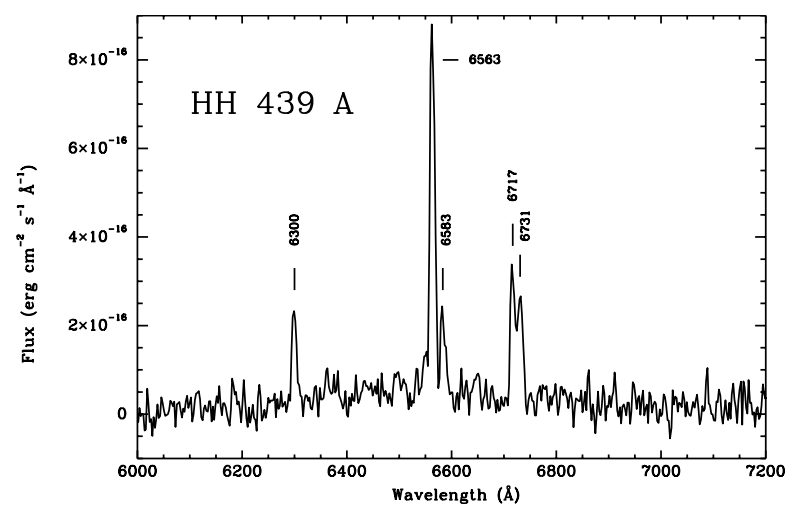

Fig. 5. Low dispersion spectrum of HH 439 A.

to its west. $\mathrm{An}_{\alpha}$ emission star nearby to $\mathrm{HH} 182$ was found in a survey of the Orion cloud (Wiramihardja et al. 1989). This star, named as Kiso A-0904 234, is located 3.4 southwest of HH 182. No other YSOs have been found in the region around HH 182. Within a radius of $10^{\prime}$, there are three IRAS sources, $05513+0236,05518+0243$, and $05515+0227$. The far-infrared 


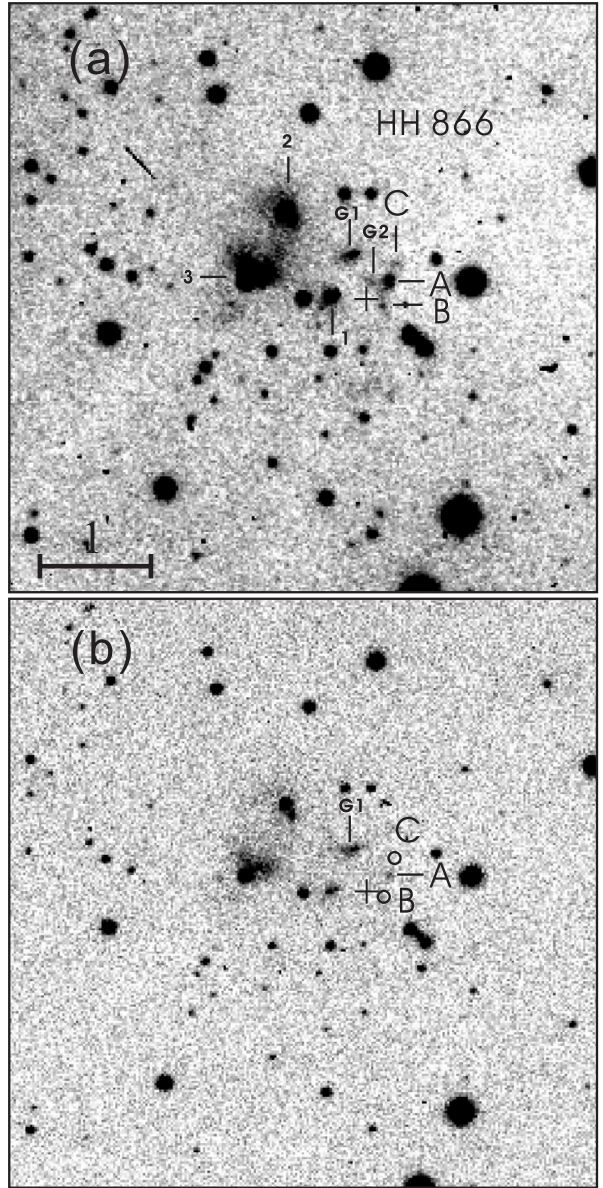

Fig. 6. Optical images of HH 866 taken with the TLS Schmidt telescope, a) in the [S II] band; b) in the $\mathrm{H}_{\alpha}$ band. The location of $\mathrm{HH} 866 \mathrm{~B}$ and $\mathrm{C}$ in the $\mathrm{H}_{\alpha}$ image is marked with open circles. The nominal position of IRAS 06046-0603 is marked with a plus sign. The extended features in the vicinity of $\mathrm{HH} 866$, designated G1 and G2, are indicated in a). These features are not $\mathrm{HH}$ objects (see Sect. 4.3 for a discussion of their nature). Stars $1-3$, which are surrounded by substantial nebulosity especially in the [S II] image, are indicated in a). Others are the same as in Fig. 4.

colors of these sources do not suggest that they are YSOs (see, e.g., Harris et al. 1988; Beichman et al. 1986).

In order to search for the exciting source of $\mathrm{HH} 182$, we carried out near-infrared $J H K^{\prime}$ imaging towards the $\mathrm{HH} 182$ region. The $J H K^{\prime}$ composite image of the region is presented in Fig. 7. Our detection limits in the $J, H$, and $K^{\prime}$ bands are estimated to be around 19.3, 17.4, and 16.0 mag, respectively. From our photometry no near-infrared excess stars are found nearby to $\mathrm{HH}$ 182. The $\mathrm{H}_{\alpha}$ emission star Kiso A-0904 234, with a photometry of $10.9,9.7$, and $9.2 \mathrm{mag}$ in the $J, H$, and $K^{\prime}$ bands, is relatively bright in the near-infrared compared with its visual magnitude ( $v=18.6 \mathrm{mag}$, Wiramihardja et al. 1989). However, this star does not show near-infrared emission excess, therefore, may not be the exciting source of HH 182.

Reipurth et al. (1997a) have shown that the HH 111 flow, consisting of $\mathrm{HH} 111,113$ and 311 , is a giant flow with an angular size of $1^{\circ}$. The HH 111 flow lies nearly in the plane of sky with a PA of around $100^{\circ}$. HH 182 is located 10 ' 6 southeast of $\mathrm{HH} 113$ in the direction of $\mathrm{PA} \simeq 125^{\circ}$. A sketch of the

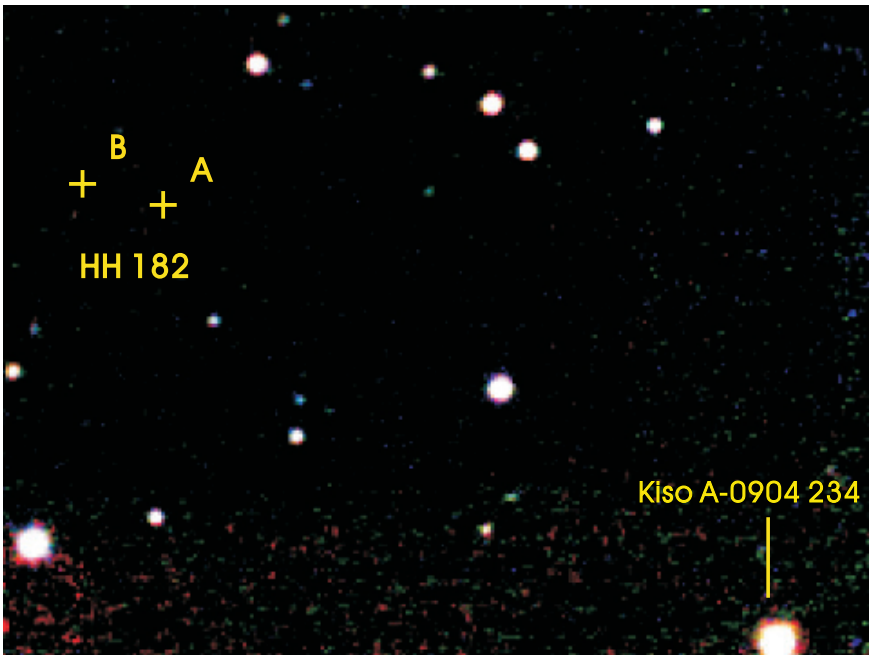

Fig. 7. Composite $J H K^{\prime}$ image of the HH 182 region. The field is $3 ! 9 \times 2$ ! 9 . Positions of HH $182 \mathrm{~A}$ and B are indicated with plus signs. North is up, east is to the left.

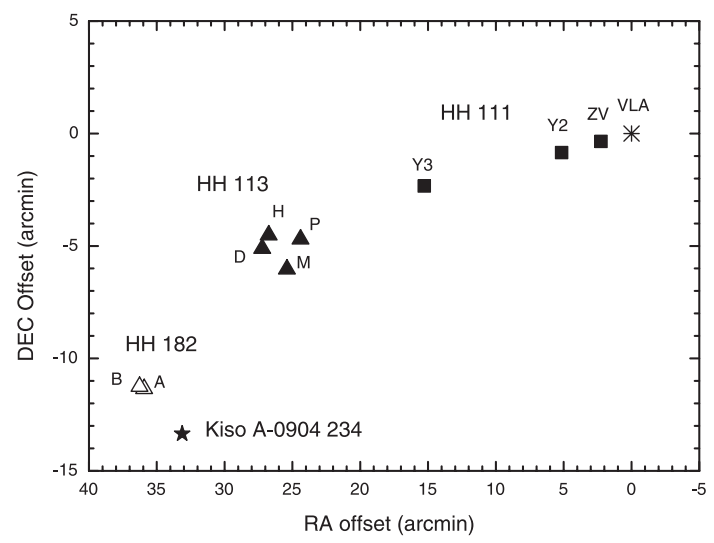

Fig. 8. A sketch of the relative positions of the inner southeastern part of the giant HH 111 flow (HH $111 \mathrm{ZV}, \mathrm{Y} 2$, and Y3, filled squares), the outer part $\mathrm{HH} 113$ (filled triangles), and the newly discovered HH 182 A and B (open triangles), with respect to the HH 111 driving source VLA (asterisk). The location of the $\mathrm{H}_{\alpha}$ emission star Kiso A-0904 234 is also indicated.

relative positions of the southeastern part of $\mathrm{HH} 111, \mathrm{HH} 113$ and $\mathrm{HH} 182$, with respect to the $\mathrm{HH} 111$ driving source, is given in Fig. 8. The position angle of $\mathrm{HH} 182$ with respect to the exciting source of the $\mathrm{HH} 111$ flow is $107^{\circ}$ and the separation is $38^{\prime}$. We note that the inner southeastern part of the $\mathrm{HH} 111$ flow, i.e. from the exciting source to knot Y3, has a position angle of $98^{\circ}$ and an angular length of $15^{\prime}$, while the outer part represented by HH 113 has a position angle of $101^{\circ}$ and a separation of $28^{\prime}$. The position angles of the inner and outer southeastern parts of the $\mathrm{HH} 111$ flow and that of $\mathrm{HH}$ 182 increase consistently with the separation from the exciting source of the HH 111 flow, suggesting that HH 182 may represent the outermost part of the southeastern lobe of the HH 111 giant flow. Proper motion observations of HH 182 can provide a critical test of this possibility. If $\mathrm{HH} 182$ is indeed part of the HH 111 flow, the scale of this flow then amounts to $1.2^{\circ}$, corresponding to a linear scale of $9.6 \mathrm{pc}$ at the distance of $460 \mathrm{pc}$. 


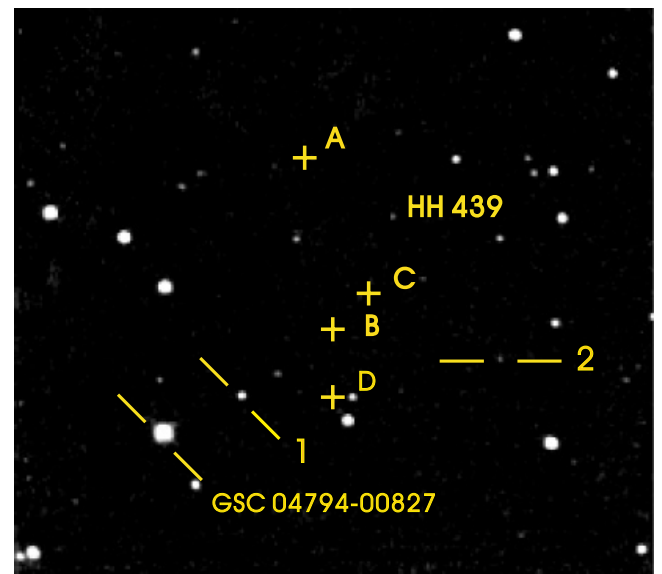

Fig. 9. Composite $J H K^{\prime}$ image of the $H H 439$ region. The field is 3. $9 \times 3$ '.5. Positions of HH 439 A-D are indicated with plus signs. North is up, east is to the left.

From Fig. $3 b$ it can be seen that the near-infrared $\mathrm{H}_{2}$ emission of $\mathrm{HH} 182 \mathrm{~A}$ is shifted towards the east with respect to the optical emission. The separation between the optical and the $\mathrm{H}_{2}$ emission is $\sim 3^{\prime \prime} .5$, corresponding to a linear separation of $1600 \mathrm{AU}$ at the distance of $460 \mathrm{pc}$. A configuration of an $\mathrm{H}_{2}$ emission arc slightly preceding the optical emission can be produced by magnetic precursors (Draine 1980). In this $\mathrm{H}_{2}$ excitation scenario, $\mathrm{H}_{2}$ emission lies slightly ahead of the optical emission. The optical and infrared observations of $\mathrm{HH} 7$ match well the predictions of $\mathrm{H}_{2}$ emission excited by magnetic precursors (Carr 1993). Therefore, HH 182 may be another good example of molecular hydrogen excitation by magnetic precursors if it is part of the giant HH 111 flow or if it is driven by a source located to its west.

\section{2. $\mathrm{HH} 439$}

$\mathrm{HH} 439$ is associated with a Herbig AeBe star candidate, GSC 04794-00827 (IRAS 06045-0554) (see Fig. 4a), which shows $\mathrm{H}_{\alpha}$ and [O I] emission (Torres et al. 1995; Vieira et al. 2003). This star is nebulous in our [S II] and $\mathrm{H}_{\alpha}$ images. A nebulosity tail extending to its southeast can be seen in our [S II] image. This emission tail may trace an outflow from the star. HH 439 is located on the opposite side of this star, therefore, it may be driven by the young star GSC 0479400827. However, the bow shock structure seen in Fig. 4a is not consistent with the identification of this star as the driving source.

The $J H K^{\prime}$ composite image of the $\mathrm{HH} 439$ region is presented in Fig. 9. From our $J H K^{\prime}$ photometry, three stars, GSC 04794-00827, 1 and 2 (see Fig. 9), show a nearinfrared excess, among them star 2 has significant nearinfrared excess (see Fig. 10). The near-infrared colors of star GSC 04794-00827 are consistent with its identification as an Herbig AeBe star candidate. The deeply embedded star 2 is located on the axis of the bow shock emission which faces star 2 (see Fig. 4a). This configuration suggests that the bow shock emission, which consists of $\mathrm{HH} 439$ A and the diffuse emission to its east, is excited by the deeply embedded star 2 .

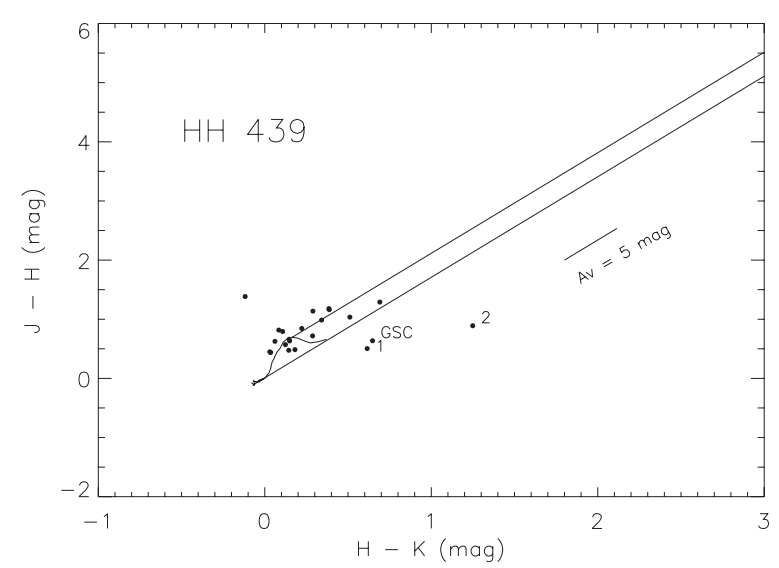

Fig. 10. Near-infrared color-color digram of stars in the HH 439 region. The curve represents the intrinsic colors of main-sequence stars (Bessell \& Brett 1988; Wegner 1994). The zone between the two straight lines is the near-infrared color regime of main-sequence stars with interstellar extinction. The extinction law is taken from Rieke \& Lebofsky (1985). A reddening vector of $A v=5$ mag is shown. Stars 1 , 2 and GSC 04794-00827 (see Fig. 9) have near-infrared color excess and are indicated. The star GSC 04794-00827 is marked "GSC" for clarity.

However, it is difficult to attribute all the emission features including HH 439 B-D to star 2, as the outflow opening angle will be too large. It is possible that $\mathrm{HH} 439 \mathrm{~A}-\mathrm{D}$ trace more than one flow. We suggest that $\mathrm{HH} 439 \mathrm{~A}$ and the associated bow shock are excited by the deeply embedded star 2 and that HH 439 B-D are excited by the young star GSC 04794-00827. However, on the basis of alignment only, we cannot completely exclude the possibility that star 1 is the driving source of features B and C. In addition, the nebula around star GSC 0479400827 seems to be elongated along an axis not coincident with the $\mathrm{B}-\mathrm{C}$ features.

Within a radius of $10^{\prime}$ from $\mathrm{HH} 439$, there are two IRAS sources, 06045-0554 (GSC 04794-00827) and 06047-0546. IRAS 06047-0546, with fluxes of 0.25, 1.66, 4.83 and $3.95 \mathrm{Jy}$ in the $12,25,60$ and $100 \mu \mathrm{m}$ bands, has farinfrared colors of embedded YSOs (Beichman et al. 1986). It is located 7.4 to the northeast of $\mathrm{HH} 439 \mathrm{~A}$ in the direction of $\mathrm{PA} \sim 35^{\circ}$. This source is outside the field of view of our near infrared imaging. It is not detected by $2 \mathrm{MASS}$, indicating that it is a deeply embedded source. An X-ray source, RX J0607.30549 (Gregorio-Hetem et al. 1998), is located 7.5 northeast of $\mathrm{HH} 439 \mathrm{~A}$ in the direction of $\mathrm{PA} \simeq 60^{\circ}$. These sources, however, are unlikely to be the driving sources of HH 439.

\section{3. $\mathrm{HH} 866$}

HH 866, located $10^{\prime} .6$ to the southeast of the newly discovered $\mathrm{HH}$ object $\mathrm{HH} 439$, is closely associated with IRAS 06046-0603 (see Fig. 6a). In the vicinity of IRAS 060460603, an infrared cluster of 15 member stars has been found (Carpenter 2000). The far-infrared colors of IRAS 06046-0603 show that it is an embedded source (Carballo \& Sahu 1994). CO line $(J=1-0)$ observations suggest that IRAS 06046-0603 is an outflow source candidate (Xu et al. 2001). Moreover, 
the configuration of $\mathrm{HH} 866 \mathrm{~A}-\mathrm{C}$ also suggests that they are driven by IRAS 06046-0603. Therefore, we identify IRAS 06046-0603 as the driving source of HH 866.

To the northeast and east of HH $866 \mathrm{~A}$, two extended features can be identified in the [S II] image. These features are designated as G1 and G2 and are marked in Fig. 6a. These features also appear in the $I$ band image (not presented in the paper), therefore, they are not HH objects. Both of them are detected by 2MASS. Feature G1 corresponds to 2MASX J06070873-0603212 and is identified as a galaxy in the 2MASS catalogue. Feature G2 corresponds to 2MASX J06070812-0603352. We note that feature G2 is much brighter in the 2MASS $H$ and $K$ images than in the 2MASS $J$ image. This feature may be a galaxy or a reflection nebula. In Fig. 6, stars 1-3 are surrounded by substantial nebulosity especially in the $[\mathrm{S} \mathrm{II}]$ image. However, these stars are not known emission line stars.

\section{Summary and conclusions}

We carried out a wide field survey of HH objects towards the L1617 and L1646 dark clouds. The results and conclusions are summarized as follows:

1. In the L1617 dark cloud one new HH object, HH 182, has been discovered. The HH object nature of HH $182 \mathrm{~A}$ has been confirmed by optical spectroscopy. Near-infrared imaging reveals three $\mathrm{H}_{2}$ emission features in the region which coincide with optical emission. Based on the position angles of the different parts of the HH 111 flow and that of $\mathrm{HH} 182$, HH 182 may be the outermost southeastern part of the giant $\mathrm{HH} 111$ flow.

2. Two new HH objects, HH 439 and 866, were discovered in the L1646 dark cloud. One deeply embedded star was revealed in our near-infrared imaging of the $\mathrm{HH} 439$ region. HH $439 \mathrm{~A}$ and the associated bow shock are probably driven by the newly detected embedded star. HH 439 B-D are probably driven by the Herbig AeBe star candidate GSC 04794-00827 (IRAS 06045-0554). HH 866 is probably driven by the embedded source IRAS 06046-0603.

Acknowledgements. We thank the staff members of BATC Beijing group, the staff members of Okayama Astronomical Observatory, Japan and the staff members of BAO for their excellent support during the observations used in this paper. We thank Jun Yan and Min Wang for their help during the BAO optical imaging observations and Yongqiang Yao and Zhibo Jiang for their help during the near infrared observations. H. Wang acknowledges the support by NSFC grants 10243004 and 10073021.

\section{References}

Bachiller, R. 1996, ARA\&A, 34, 111

Bally, J., Langer, W. D., Wilson, R. W., et al. 1991, Fragmentation of Molecular Clouds and Star Formation, ed. E. Falgarone, F. Boulanger, \& G. Duvert (Kluwer), IAU Symp., 147, 11

Bacciotti, F., Chiuderi, C., \& Oliva, E. 1995, A\&A, 296, 185
Beichman, C. A., Myers, P. C., Emerson, J. P., et al. 1986, ApJ, 307, 337

Bessell, M. S., \& Brett, J. M. 1988, PASP, 100, 1134

Carballo, R., \& Eiroa, C. 1992, A\&A, 262, 295

Carballo, R., \& Sahu, M. 1994, A\&A, 289, 131

Carpenter, J. M. 2000, AJ, 120, 3139

Carr, J. 1993, ApJ, 406, 553

Dent, W. R. F., Matthews, H. E., \& Ward-Thompson, D. 1998, MNRAS, 301, 1049

Devine, D., Bally, J., Reipurth, B., \& Heathcote, S. 1997, AJ, 114, 2095

Draine, B. 1980, ApJ, 241, 1021

Eislöffel, J. 1997, IAU Symp. (Kluwer Academic Publisher), 182, 93

Eislöffel, J., \& Mundt, R. 1997, AJ, 114, 280

Fan, X., Burstein, D., Chen, J., et al. 1996, AJ, 112, 628

Gredel, R., \& Reipurth, B. 1993, ApJ, 407, L29

Gregorio-Hetem, J., Montmerle, T., Casanova, S., \& Feigelson, E. D. 1998, A\&A, 331, 193

Harris, S., Clegg, P., \& Hughes, J. 1988, MNRAS, 235, 441

Hartigan, P., Bally, J., et al. 2000, Protostars and Planets IV, 841

Hartigan, P., Morse, J., \& Raymond, J. 1994, ApJ, 436, 125

Herbig, G. H., \& Bell, K. R. 1988, Lick Obs. Bull. No. 1111

Herbst, W., \& Racine, R. 1976, AJ, 81, 840

Hunt, L. K., Mannucci, F., Testi, L., et al. 1998, AJ, 115, 2594

Königl, A., \& Pudritz, R. E. 2000, in Protostars and Planets IV, ed. V. Mannings, A. P. Boss, \& S. S. Russell (Tucson: Univ. Arizona Press), 759

Noriega-Crespo, A., Garnavich, P. M., Moro-Martin, A., et al. 2002, BAAS, 201, 142.02

Pravdo, S. H., Rodriguez, L. F., Curiel, S., et al. 1985, ApJ, 293, L35

Osterbrock, D. E. 1989, Astrophysics of Gaseous Nebulae and Active Galactic Nuclei (Mill Vally: University Science Books)

Raga, A. C., Böhm, K. H., \& Cantó, J. 1996, Rev. Mex. Astron. Astrofis., 32, 161

Reipurth, B., \& Olberg, M. 1991, A\&A, 246, 535

Reipurth, B., Chini, R., Krügel, E., Kreysa, E., \& Sievers, A. 1993 , A\&A, 273, 221

Reipurth, B., Raga, A. C., \& Heathcote, S. 1996, A\&A, 311, 989

Reipurth, B., Bally, J., \& Devine, D. 1997a, AJ, 114, 2708

Reipurth, B., Hartigan, P., Heathcote, S., Morse, J., \& Bally, J. 1997b, AJ, 114, 757

Rieke, G. H., \& Lebofsky, M. J. 1985, ApJ, 288, 618

Rodríguez, L. F., Reipurth, B., Raga, A. C., \& Cantó, J., 1998, Rev. Mex. Astron. Astrofis., 34, 69

Shu, F. H., Najita, J. R., Shang, H., \& Li, Z.-Y. 2000, in Protostars and Planets IV, ed. V. Mannings, A. P. Boss, \& S. S. Russell (Tucson: Univ. Arizona Press), 789

Torres, C. A. O., Quast, G., de La Reza, R., Gregorio-Hetem, J., \& Lepine, J. R. D. 1995, AJ, 109, 2146

Wang, H., Yang, J., Wang, M., et al. 2001, AJ, 121, 1551

Wang, H., Yang, J., Wang, M., \& Yan, J. 2003, AJ, 125, 842

Wiramihardja, S. D., Kogure, T., Yoshida, S., Ogura, K., \& Nakano, M. 1989, PASJ, 41, 155

Yan, J., Wang, H., Wang, M., et al. 1998, AJ, 116, 2438

Yamashita, T., Nishihara, E., Okumura, S., Mori, A., \& Watanabe, E. 1995, in Scientific and Engineering Frontiers for $8-10 \mathrm{~m}$ Telescopes, ed. M. Iye, \& T. Nishimura (Tokyo: Univ. Acad.), 285

Vieira, S. L. A., Corradi, W. J. B., Alencar, S. H. P., et al. 2003, AJ, 126, 2971

Wegner, W. 1994, MNRAS, 270, 229

Xu, Y., Jiang, D.-R., Yu, Z.-Y., et al. 2001, ChJAA, 1, 60

Zinnecker, H., McCaughrean, M. J., \& Rayner, J. T. 1998, Nature, 394,862 